\title{
Trustworthy guidelines on severe asthma thanks to the ERS and ATS
}

\author{
Guy G. Brusselle $e^{1,2}$ and Monica Kraft ${ }^{3}$
}

\begin{abstract}
Affiliations: ${ }^{1}$ Dept of Respiratory Medicine, Ghent University Hospital, Ghent, Belgium. ${ }^{2}$ Depts of Epidemiology and Respiratory Medicine, Erasmus Medical Center, Rotterdam, The Netherlands. ${ }^{3}$ Division of Pulmonary, Allergy and Critical Care Medicine, Duke University Medical Centre, Durham, NC, USA.
\end{abstract}

Correspondence: G.G. Brusselle, Dept of Respiratory Medicine, Ghent University Hospital, De Pintelaan 185, 7K12, 9000 Ghent, Belgium. E-mail: guy.brusselledugent.be

0

$@$ ERSpublications

Implementation of the ERS/ATS guidelines on severe asthma will ultimately improve the health of our patients http://ow.ly/rgnrM

\begin{abstract}
Although severe asthma is estimated to be present in less than $10 \%$ of all asthmatics, these patients have the greatest morbidity and consume an overwhelming proportion of healthcare costs [1]. There have also been challenges defining the disease in terms of severity and control, characteristics which both lead to increased morbidity and mortality. Thus, the greatest unmet need in asthma care is in the severe asthma arena, where heterogeneity with regard to clinical presentation and course has posed therapeutic challenges. The identification of clinical and molecular phenotypes, as discussed in this version of the severe asthma guidelines, moves the field forward and will ultimately lead us to more personalised therapy. These guidelines describe the definition of severe asthma and provide recommendations for an approach to diagnostics and therapeutics given the data available today.
\end{abstract}

Clinical practice guidelines aim to help clinicians and healthcare professionals to make evidence-based decisions about the optimal care for patients [2]. In the past decade, major progress has been made in the methodology and the science of developing guidelines [3]. In contrast to narrative reviews or expert consensus-based clinical position statements (also called "strategic documents"), the development of guidelines requires that the evidence is appraised in a comprehensive and systematic manner and that the recommendations for practice are provided in a transparent manner [3, 4]. The Institute of Medicine has published standards for trustworthy guidelines, providing recommendations on several crucial domains in the development process including transparency, panel composition and conflict of interest [4-6]. Importantly, funders should not play a role in the development, and guideline panels should be multidisciplinary and include patients and/or patient advocates [6,7]. For the literature review and the grading of recommendations, a systematic approach is mandatory to rate the quality of the evidence, to summarise the benefits and harms of a given treatment, and to grade the strength of the recommendations [3]. Since the European Respiratory Society (ERS) and American Thoracic Society (ATS) have adopted the Grading of Recommendations, Assessment, Development and Evaluation (GRADE) system for these methodological requirements, the ERS/ATS Task Force has succeeded in developing trustworthy, highquality guidelines on the definition, evaluation and treatment of severe asthma, published in this issue of the European Respiratory Journal [8]. In addition, the ERS/ATS guidelines on severe asthma have many assets.

A first asset of the severe asthma guidelines is a clear definition of severe asthma and uncontrolled asthma. When a diagnosis of asthma is confirmed and comorbidities addressed, severe asthma is defined as "asthma which requires treatment with high dose inhaled corticosteroids (ICS) ... plus a second controller [long acting $\beta 2$-agonist (LABA), leukotriene modifier, theophylline] (and/or systemic corticosteroids) to prevent it from becoming 'uncontrolled' or which remains 'uncontrolled' despite this therapy". The updated

Received: Nov 042013 | Accepted after revision: Nov 142013

Conflict of interest: Disclosures can be found alongside the online version of this article at www.erj.ersjournals.com

Copyright (CERS 2014 
ERS/ATS Task Force definition, thus, focuses on severe asthma refractory to currently available medications using a simple definition that can be translated to clinical practice, in contrast to untreated severe asthma (as used previously in the first and second version of the Global Initiative for Asthma (GINA) guidelines) $[9,10]$. Uncontrolled asthma is defined by the presence of any one of four criteria, encompassing: 1) poor symptom control, 2) frequent severe exacerbations, 3) serious exacerbations and 4) airflow limitation. This definition underlines the heterogeneity of uncontrolled severe asthma, since both patients with persistent airflow limitation and/or repetitive exacerbations qualify as uncontrolled asthmatics [11, 12]. A similar classification approach has been used by the Global Initiative for Chronic Obstructive Lung disease (GOLD), since the updated strategic document qualifies chronic obstructive pulmonary disease (COPD) patients into the most severe category (GOLD category D) based on the presence of low lung function and/ or frequent exacerbations [13].

A second asset of the ERS/ATS guidelines is the detailed chapter on phenotyping of severe asthma, highlighting that severe asthma is a heterogeneous syndrome. Phenotyping integrates biological and clinical features and aims to improve therapy (by predicting the therapeutic response to available drugs in prespecified phenotypes or by identifying novel therapeutic targets in specific phenotypes) [14]. Important clinical features in phenotyping severe asthma are the age at onset of the disease, the presence or absence of allergy, the frequency of exacerbations and the level of airflow limitation [11, 12, 14]. An important biological feature is the nature of the underlying airway inflammation, discerning eosinophilic and noneosinophilic (neutrophilic or paucigranulocytic) severe asthma $[14,15]$. While nitric oxide is currently not recommended to guide therapy in adults and children with severe asthma, it may be valuable to determine the inflammatory phenotype and decisions regarding the use of T-helper cell (Th) 2 focused biological therapy [16-18]. The crucial role of asthma phenotyping is well illustrated by the history of the clinical development of monoclonal antibodies against interleukin (IL)-5 (mepolizumab and reslizumab). In the original studies, anti-IL5 monoclonal antibodies were used as add-on therapy in patients with moderate-tosevere asthma, without phenotyping the underlying airway inflammation, and the effect on lung function was evaluated as the primary outcome $[19,20]$. These studies were negative. In contrast, when only patients with severe asthma and evidence of persistent eosinophilic airway inflammation were enrolled add-on therapy with anti-IL5 monoclonal antibodies, on top of ICS and LABA, was shown to significantly reduce the rate of moderate and severe exacerbations [21-23]. Similarly, most randomised controlled trials (RCTs) of macrolide antibiotics did not show benefits in patients with moderate-to-severe asthma, explaining the sixth recommendation of the ERS/ATS guidelines [24-26]. However, a recent trial of long-term treatment with azithromycin in severe asthma suggests that patients with exacerbation-prone, non-eosinophilic asthma might benefit [27]. Although this hypothesis has to be confirmed in larger RCTs, the observations are in line with the proven efficacy of macrolides in other neutrophilic chronic airway diseases, including cystic fibrosis (CF), non-CF bronchiectasis and diffuse panbronchiolitis [28-31].

In addition to phenotype specific therapies, new therapies such as bronchial thermoplasty, have also been included for discussion and evaluation in this guideline. Bronchial thermoplasty is a unique, new therapy that employs radiofrequency heat applied directly to the airways as treatment for severe asthma [32]. There are data to suggest the effects are sustained, but the asthmatic patients who received sham bronchoscopy were not followed long term [33]. The technique did reveal improvements in severe exacerbations, an outcome that is extremely important in severe asthma (Asthma Intervention Research (AIR)2 trial) [34]. However, until there are data that allow us to identify the best candidates for this expensive and invasive procedure, the recommendation for additional study and use in specialised centres put forth in this guideline is reasonable. There is considerable interest in studying this therapy within the asthma community and, hopefully, adding it to our armamentarium, as effective treatment for severe asthma is an unmet need. However, in an era of phenotype-driven therapy, bronchial thermoplasty should not be an exception.

Furthermore, the ERS/ATS Task Force has not only taken into account evidence on efficacy and safety of drugs in severe asthma from RCTs, but also complementary evidence on real-life effectiveness, costeffectiveness and long-term safety from observational studies. While classical RCTs provide the highest quality evidence, the outcomes reported from RCTs may not always be generalisable to real-world patients followed in clinical practice settings $[35,36]$. Indeed, classical RCTs, including tightly controlled registration trials, investigate the effects of a new drug, compared with placebo, in specialised centres on surrogate outcomes (such as lung function) in highly selected patients, without comorbidities but with optimal adherence to the study treatment and protocol. The ERS/ATS Workshop Report on Guideline Development [35] as well as the respiratory effectiveness group (www.effectivenessevaluation.org) plea that guideline developers should also consider evidence from pragmatic trials and nonrandomised studies, investigating the real-life effectiveness and long-term safety of a treatment in large populations of heterogeneous patients followed in routine care. Since real-life patients often have comorbid illnesses and lower adherence to medications it is important to complement the efficacy data from RCTs, obtained in ideal circumstances, 
with effectiveness data from observational studies. The paucity of real-life effectiveness and costeffectiveness data in severe asthma has contributed to the fact that the ERS/ATS Task Force has graded the quality of the evidence of most of the specific clinical recommendations on the treatment of severe asthma as "low" or "very low" and the strength of the respective recommendations as "conditional".

As the field of severe asthma is dynamic and not all areas are well supported by significant evidence in 2013, there are limitations to these guidelines. This is reflected in the relatively limited number of PICO (Patients/ Intervention/Comparator/Outcome) questions which could be addressed by the ERS/ATS Task Force. This observation suggests that there must be updates to include the latest literature as the field moves forward to create a "living document". Examples of areas to expand upon in the future include: the role of long-acting muscarinic antagonists in severe asthma; the role of monoclonal antibodies targeting specific cytokines; how exhaled nitric oxide is best used in clinical practice; and issues surrounding asthma pathobiology such as innate immune mechanisms, airway remodelling and the microbiome.

What we can do today is ensure that these guidelines are disseminated widely through our societies, through thoughtful, coordinated action at our annual scientific meetings, and also through our journals, websites and other modes of communication such as webinars, podcasts and toolkits for providers and patients. We have a unique opportunity not only to disseminate, but to implement these guidelines to ultimately improve the health of our patients with severe asthma.

\section{References}

1 Moore WC, Bleecker ER, Curran-Everett D, et al. Characterization of the severe asthma phenotype by the National Heart, Lung, and Blood Institute's Severe Asthma Research Program. J Allergy Clin Immunol 2007; 119: 405-413.

2 Schünemann HJ, Woodhead M, Anzueto A, et al. A guide to guidelines for professional societies and other developers of recommendations: introduction to integrating and coordinating efforts in COPD guideline development. An official ATS/ERS workshop report. Proc Am Thorac Soc 2012; 9: 215-218.

3 Brozek JL, Akl EA, Jaeschke R, et al. Grading quality of evidence and strength of recommendations in clinical practice guidelines: part 2 of 3 . The GRADE approach to grading quality of evidence about diagnostic tests and strategies. Allergy 2009; 64: 1109-1116.

4 Kuehn BM. IOM sets out "gold standard" practices for creating guidelines, systematic reviews. JAMA 2011; 305: $1846-1848$.

5 Kunz R, Fretheim A, Cluzeau F, et al. Guideline group composition and group processes: article 3 in Integrating and coordinating efforts in COPD guideline development. An official ATS/ERS workshop report. Proc Am Thorac Soc 2012; 9: 229-233.

6 Boyd EA, Akl EA, Baumann M, et al. Guideline funding and conflicts of interest: article 4 in Integrating and coordinating efforts in COPD guideline development. An official ATS/ERS workshop report. Proc Am Thorac Soc 2012; 9: 234-242.

7 Kelson M, Akl EA, Bastian $\mathrm{H}$, et al. Integrating values and consumer involvement in guidelines with the patient at the center: article 8 in Integrating and coordinating efforts in COPD guideline development. An official ATS/ERS workshop report. Proc Am Thorac Soc 2012; 9: 262-268.

8 Chung KF, Wenzel SE, Brozek JL, et al. International ERS/ATS guidelines on definition, evaluation and treatment of severe asthma. Eur Respir J 2014; 43: 343-373.

9 NHLBI/WHO Workshop Report: Global Strategy for Asthma Management and Prevention. Bethesda, National Institutes of Health, National Heart, Lung and Blood Institute, 1995.

10 Global Initiative for Asthma. Global Strategy for Asthma Management and Prevention. Bethesda, National Institutes of Health, National Heart, Lung and Blood Institute, 2002.

11 Wenzel SE. Asthma: defining of the persistent adult phenotypes. Lancet 2006; 368: 804-813.

12 Moore WC, Meyers DA, Wenzel SE, et al. Identification of asthma phenotypes using cluster analysis in the Severe Asthma Research Program. Am J Respir Crit Care Med 2010; 181: 315-323.

13 Vestbo J, Hurd SS, Agustí AG, et al. Global strategy for the diagnosis, management and prevention of chronic obstructive pulmonary disease: GOLD executive summary. Am J Respir Crit Care Med 2013; 187: 347-365.

14 Wenzel SE. Asthma phenotypes: the evolution from clinical to molecular approaches. Nat Med 2012; 18: 716-725.

15 Haldar P, Pavord ID, Shaw DE, et al. Cluster analysis and clinical asthma phenotypes. Am J Respir Crit Care Med 2008; 178: 218-224.

16 Corren J, Lemanske RF, Hanania NA, et al. Lebrikizumab treatment in adults with asthma. N Engl J Med 2011;365: $1088-1098$.

17 Piper E, Brightling C, Niven R, et al. A phase II placebo-controlled study of tralokinumab in moderate-to-severe asthma. Eur Respir J 2013; 41: 330-338.

18 Corren J. Anti-interleukin-13 antibody therapy for asthma: one step closer. Eur Respir J 2013; 41: 255-256.

19 Leckie MJ, ten Brinke A, Khan J, et al. Effects of an interleukin-5 blocking monoclonal antibody on eosinophils, airway hyper-responsiveness, and the late asthmatic response. Lancet 2000; 356: 2144-2148.

20 Flood-Page P, Swenson C, Faiferman I, et al. A study to evaluate safety and efficacy of mepolizumab in patients with moderate persistent asthma. Am J Respir Crit Care Med 2007; 176: 1062-1071.

21 Haldar P, Brightling C, Hargadon B, et al. Mepolizumab and exacerbations of refractory eosinophilic asthma. N Engl J Med 2009; 360: 973-984.

22 Nair P, Pizzichini M, Kjarsgaard M, et al. Mepolizumab for prednisone-dependent asthma with sputum eosinophilia. N Engl J Med 2009; 360: 985-993.

23 Pavord ID, Korn S, Howarth P, et al. Mepolizumab for severe eosinophilic asthma (DREAM): a multicentre, double-blind, placebo-controlled trial. Lancet 2012; 380: 651-659. 
24 Black PN, Blasi F, Jenkins CR, et al. Trial of roxithromycin in subjects with asthma and serological evidence of infection with Chlamydia pneumoniae. Am J Respir Crit Care Med 2001; 164: 536-541.

25 Richeldi L, Ferrara G, Fabbri LM, et al. Macrolides for chronic asthma. Cochrane Database Syst Rev 2005; 4: CD002997.

26 Cameron EJ, Chaudhuri R, Mair F, et al. Randomised controlled trial of azithromycin in smokers with asthma. Eur Respir J 2013; 42: 1412-1415.

27 Brusselle GG, Vanderstichele C, Jordens P, et al. Azithromycin for prevention of exacerbations in severe asthma (AZISAST): a multicentre randomised double-blind placebo-controlled trial. Thorax 2013; 68: 322-329.

28 Equi A, Balfour-Lynn IM, Bush A, et al. Long term azithromycin in children with cystic fibrosis: a randomised, placebo-controlled crossover trial. Lancet 2002; 360: 978-984.

29 Altenburg J, de Graaff CS, Stienstra Y, et al. Effect of azithromycin maintenance treatment on infectious exacerbations among patients with non-cystic fibrosis bronchiectasis: the BAT randomized controlled trial. JAMA 2013; 309: 1251-1259.

30 Koyama H, Geddes DM. Erythromycin and diffuse panbronchiolitis. Thorax 1997; 52: 915-918.

31 Wong C, Jayaram L, Karalus N, et al. Azithromycin for prevention of exacerbations in non-cystic fibrosis bronchiectasis (EMBRACE): a randomised, double-blind, placebo-controlled trial. Lancet 2012; 380: 660-667.

32 Wahidi MM, Kraft M. Bronchial thermoplasty for severe asthma. Am J Respir Crit Care Med 2012; 185: 709-714.

33 Wechsler ME, Laviolette M, Rubin AS, et al. Bronchial thermoplasty: long-term safety and effectiveness in patients with severe persistent asthma. J Allergy Clin Immunol 2013 [in press DOI: 10.1016/j.jaci.2013.08.009].

34 Castro M, Rubin AS, Laviolette M, et al. Effectiveness and safety of bronchial thermoplasty in the treatment of severe asthma: a multicenter, randomized, double-blind, sham-controlled clinical trial. Am J Respir Crit Care Med 2010; 181: 116-124.

35 Wilt T, Guyatt G, Kunz R, et al. Deciding what type of evidence and outcomes to include in guidelines: article 5 in Integrating and coordinating efforts in COPD guideline development. An official ATS/ERS workshop report. Proc Am Thorac Soc 2012; 9: 243-250.

36 Avorn J. In defense of pharmacoepidemiology - embracing the yin and yang of drug research. N Engl J Med 2007; 357: 2219-2221. 